\title{
Research on the Cooperative Behavior of Academic Papers Published by Chinese Educational Scholars Based on Complex Networks
}

\author{
Bichu Li 1,** $\&$ Ziliang Zhang $^{2}$ \\ ${ }^{1}$ Naveen Jindal School of Management, University of Texas at Dallas, Richardson, United States \\ ${ }^{2}$ Erik Jonsson School of Engineering and Computer Science, University of Texas at Dallas, Richardson, United \\ States \\ *Correspondence: Naveen Jindao School of Management, University of Texas at Dallas, Richardson, United States. \\ E-mail: bx1161130@utdallas.edu
}

Received: November 17, 2018

Accepted: January 11, 2019 Online Published: February 14, 2019

doi:10.5430/wje.v9n1p118

URL: https://doi.org/10.5430/wje.v9n1p118

\begin{abstract}
Research on mutual cooperation among scholars or research institutions has become more and more common. The purpose of this paper is to explore the current status of cooperation between scholars and research institutions in the field of Chinese education. In this paper, we use the method of the complex network to analyze the cooperative behavior of academic papers published by Chinese educational scholars by collecting academic papers on education leadership, education policy, quality education, and vocational education. Our conclusions show that most of the academic papers published by Chinese educational scholars are non-cooperative. In the authors of the co-authored papers, there is a significant "Matthew effect", that is, some key scholars in these fields that link the collaborators. Lastly, there is no obvious aggregation effect between the authors of the co-authored papers which indicating a widespread and extensive connection between the collaborators. The above conclusions provide valuable insights into our understanding of the cooperative behavior of Chinese education scholars.
\end{abstract}

Keywords: education, academic papers, cooperative behavior, complex network, key scholar

\section{Introduction}

China is a huge education country with hundreds of millions of school-age populations and a large and complete education system. Academic research in the field of education is of decisive significance for knowledge innovation, institutional innovation and personnel training in the education system. With the development of China's economy and society and science and technology, the demand for talents in the whole society has grown significantly. Meanwhile, the educational background of talents in various fields now has more detailed and targeted requirements compared with the past. As a result, pedagogy is gaining more and more attention, and there are more and more research directions in the field of pedagogy, which has led to more frequent interactions among researchers in the same field.

In the academic field, collaborative research is an important model for researchers to carry out knowledge innovation, and it is also an important way of knowledge dissemination. The social structure generated by cooperation has an important impact on the quantity and quality of knowledge production (Uzzi \& Spiro, 2005). The cooperative network structure existing in social organizations has been a subject of great concern to the behavior of organizational members and the performance of community organizations, it has been widely studied in sociology, economics, physics and other disciplines (Haifeng Nie \& Jingjun Liu, 2014). In the context of the rapid development of information technology in recent years, we can understand the complex world faster and better. Many systems in the reality that contain interactions between individuals can be abstracted into complex networks. Complex networks are one of the hotspots in the multidisciplinary research field of today's society (Watts \& Strogatz, 1998; Barabasi \& Albert, 1999). A complex network generally refers to a network with a large number of nodes and a complex connection relationship. Many things can be abstracted into complex networks in our life. For example, when we chat with friends and relatives using mobile phones, we already formed a network unconsciously and if the router is connected with multiple computers, it can also form a complex network. The structure detection of communities is 
an important research topic of complex networks. The community structure is widely used in biology, physics, computer graphics, and sociology. Moreover, many community discovery algorithms have been developed from these disciplines. Through these algorithms, we can get some well-defined community structures (Ahn et al., 2010).

This paper focuses on the field of Chinese education and studies the cooperative behavior of Chinese educational scholars publishing academic papers through complex network methods. For this purpose, we have collected and compiled academic papers published in CNKI related to education policy, education leadership, quality education, and vocational education in recent years. Through the authors and other information, we explored the relationships between authors and their organizations to understand the cooperative behavior and structural distribution characteristics of Chinese education scholars in scientific research, so that we can help promote academic innovation in China's education field and optimize resource allocation.

\section{Conceptions and Methods}

\subsection{Conceptions}

Educational Leadership: Educational leadership refers to the ability to motivate, develop, and work with each individual in a school's organization (Leithwood, Jantzi, \& Steinbach, 1999). Educational leadership focuses on leadership effectiveness, and its essence is an influence, which requires education leaders to expand ways and means to consolidate and expand their creativity, cohesion, traction, impetus, and development. Educational leadership is a key force for educational organizations to seek survival and development (Leithwood \& Riehl, 2003).

Education Policy: Educational policy is a code of conduct for education that is formulated by political parties and countries to achieve educational development goals and tasks in a certain historical period, which is based on the basic tasks and basic policies of the party and the state in a certain historical period (Bell \& Stevenson, 2006). In China, education policies include the Education Act, the Teacher's Law, the Statute of Compulsory education, the Higher Education Act, the Vocational Education Act, the Law of Generally-used Words, the Law on the Protection of Minors and many other regulations.

Quality-oriented Education: Quality-oriented education refers to an educational model that aims to improve the quality of all aspects of the educated. It attaches importance to people's ideological and moral quality, ability development, personality development, physical health, and mental health education. It aims at comprehensively improving people's basic quality, respecting human subjectivity and initiative, based on human character, focusing on developing the intellectual potential of human beings, and paying attention to the education that forms the basic characteristics of human beings' sound personality (Team, 2006).

Vocational Education: Vocational education refers to the education of the educated people to acquire the vocational knowledge, skills and professional ethics required for a certain occupation or production, such as pre-employment training for employees, reemployment training for laid-off workers, and various training. And also, vocational high school, secondary school, technical school, and other vocational school education are all included. The purpose of vocational education is to cultivate applied talents and workers with certain cultural level and professional knowledge skills (Gordon, 2003). Compared with general education and adult education, vocational education focuses on the cultivation of practical skills and practical workability.

Small-world Network: In the small world network diagram, most of the nodes are not adjacent to each other, but most of the nodes can be reached from any other point in a few steps. In this paper, each point in the small-world network is defined as the author of a paper in the direction of a particular subject, and the line that links each other represents whether the author directly has a cooperative relationship, such a small world network can respond to a certain small-world phenomenon that is connected by a cooperating author among the disciplines.

Scholars have proposed a variety of theories about the relationship between ideas and academic cooperation in sociology (Moody, 2004). One of the famous academic cooperation mechanisms is the "Matthew effect", also known as the Preferential Attachment (Merton, 1968). When a researcher exceeds other researchers due to some factors such as research ability, research status, and qualifications under certain conditions, he is more likely to receive attention, thereby attracting more resources and getting more opportunities for cooperation. Therefore, this kind of advancement makes the number of researchers' collaborators different. Some researchers have a large number of collaborators, but there may be little cooperation among their collaborators. We call these researchers 'key scholars'.

\subsection{Research Methods}

We first introduce the related concepts of the cooperation network. We use $V=\{1,2, \cdots, n\}$ to represent a 
collection of all authors in a network. For the two authors $\mathrm{i}, \mathrm{j} \in \mathrm{V}$, define the dummy variable $g_{i j}$ to indicate the cooperation relationship between them, $g_{i j}=1$ means that at least one article has been published by the two authors together, and $g_{i j}=0$ means that there is no cooperation relationship between the two. The collection of all authors and the set of cooperative academic relationships between the authors constitute the cooperative network G.

Further, we use the following equation:

$N_{i}(G)=\left\{j \in N: g_{i, j}=1\right\}$.

This equation is used to represent a collection of other authors in the cooperative network $G$ that have a cooperative relationship with the author $\mathrm{i}$. Thus, the number of collaborators of author i is $\beta_{i}(G)=\left|N_{i}(G)\right|$, which is called the degree of author $\mathrm{i}$ in network $\mathrm{G}$. The average degree of a network $\mathrm{G}$ is defined as:

$\beta(g)=\sum_{i \in V} \frac{\beta_{i}(G)}{n}$.

If $g_{i, j}=1$ or there is a set of mutually different authors $j_{1}, j_{2}, \cdots, j_{k}$ satisfying $g_{i, j_{1}}=1, g_{j_{1}, j_{i}}=1, \cdots, g_{j_{k}, j}=1$, we have a path between author $i$ and author $j$.

For the implementation of the network diagram, we use the Python packages NetworkX and Matplotlib to achieve. Python is a computer programming language. It is a dynamic, object-oriented scripting language originally designed to write automated scripts (shells). As versions continue to be updated and new language features are added, Python is increasingly being used for independent, large-scale project development. It is simple, easy to learn, fast, open source, and has a rich standard library, which is very suitable for data processing and analysis (Smedt \& Daelemans, 2012).

NetworkX is a Python package for creating, manipulating and studying the structure, dynamics, and functionality of complex networks. It has a large number of standard graph algorithms, including a wealth of network structure and analysis measures, as well as classic maps, random graphs and generators for synthetic networks. It is easy to operate and can greatly improve the speed of data analysis.

Matplotlib is the visual operator interface for the Python programming language and its numerical math extension package, NumPy. It provides an application interface to the application's embedded drawing using common graphical user interface toolkits such as Tkinter, wxPython, Qt or GTK+. In addition, Matplotlib has a Pylab interface based on the image processing library, which provides a MATLAB-like interface.

\section{Empirical Analysis}

\subsection{Data Sources and Processing}

First of all, we used python to write crawler code, and crawled the journal articles searched by "Educational Leadership", "Education Policy", "Quality Education" and "Vocational Education". We stored the title, author, author unit, abstract, citation number and other information on the articles in an excel form.

Secondly, we sort the data according to the relevance of the entry keywords and process the first 1000 pieces of the crawled data separately. We removed the entries having an invalid link, and those of which the author or the author's unit on the article's information page is empty. After that, we got the final available data, and the results are shown in Table 1.

Table 1. Number of the Related Literature, Authors and Author's Units

\begin{tabular}{lccc}
\hline Research object & $\begin{array}{c}\text { Total number of the } \\
\text { literature }\end{array}$ & $\begin{array}{c}\text { Number of entries in } \\
\text { author }\end{array}$ & $\begin{array}{c}\text { Number of entries in } \\
\text { the authoring unit }\end{array}$ \\
\hline Educational Leadership & 1015 & 1008 & 983 \\
Education Policy & 984 & 983 & 970 \\
Vocational Education & 983 & 949 & 890 \\
Quality Education & 932 & 916 & 843 \\
\hline
\end{tabular}

\subsection{Generating the Network Diagram}

We used python's existing NetworkX package to analyze the collated data and generate the corresponding relational network. Then used the Matplotlib package under Python to visualize the network, and finally got the network diagram. Figure 1 shows the cooperation network diagram based on the author's name. Each node in the network diagram represents an author, and the arrow points to the second, third, fourth author, etc. by the first author, and the 
direction of the arrow indicates the relationship between the authors of articles having multiple authors. At the same time, the nodes representing the author are weighted. The more arrows connected to one author, the more the author's collaboration with other authors, the higher the author's weight, and the larger the node represented in the network diagram as the author.

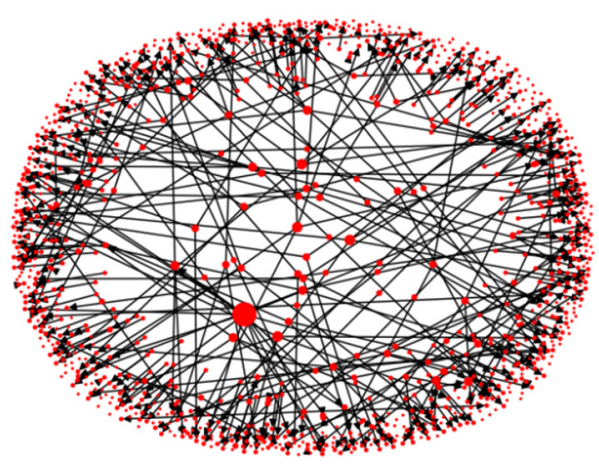

(a)

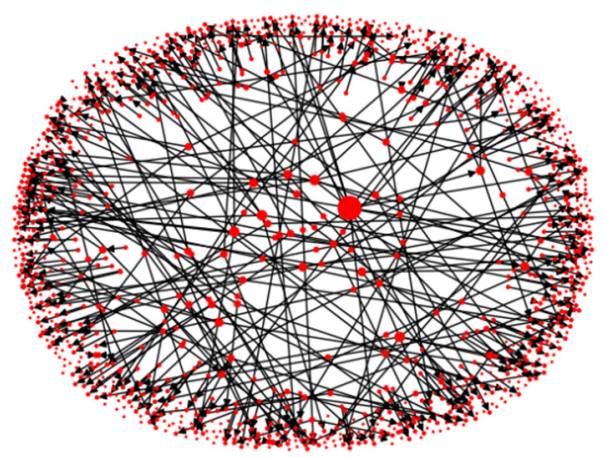

(c)

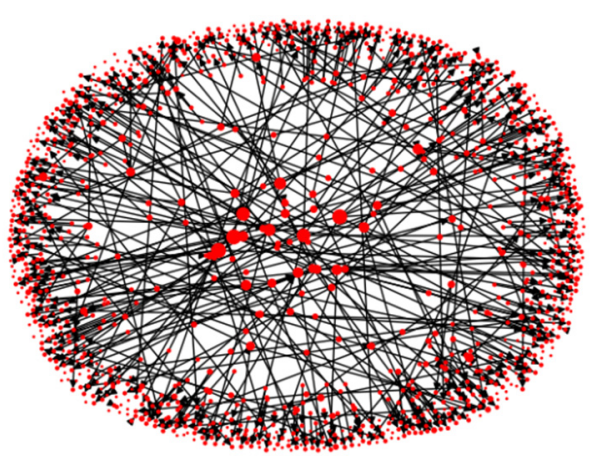

(b)

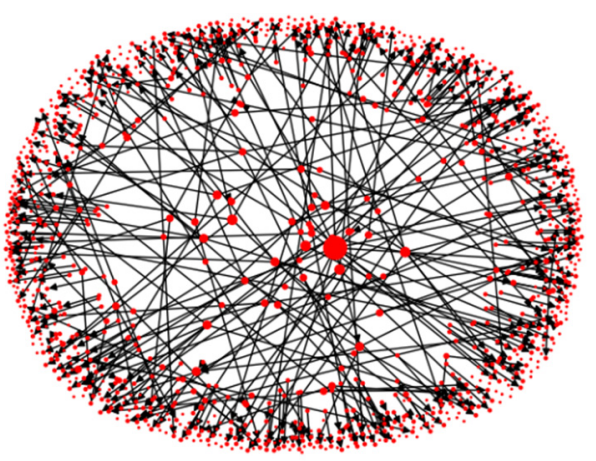

(d)

Figure 1. The Collaborative Network Based on the Author's Name

Among them, Figure (a) is the cooperation network diagram of the authors of the education leadership; Figure (b) is the cooperation network diagram of the authors of the education policy; Figure (c) is the cooperation network diagram of the authors of the quality education, and Figure (d) is a cooperative network diagram of authors of the field of vocational education

It can be seen from Figure 1 that the authors' cooperative networks based on the four key words, education leadership, education policy, quality education, and vocational education, have a similar structure. A large number of independent and uncooperative authors are scattered around the network map, forming an elliptical scatter structure. Authors of cooperative relationships form a network of reciprocal relationships to the center through directed edges. There are some large nodes in the four networks. The larger the nodes, the more the number of collaborators of the author, which forms some important authors. Among these four networks, the network structure in Figure (b) is more intensive, indicating that the number of paper collaborators on topics related to education policy is more and the degree of cooperation is more frequent. In Figures (a), (c) and (d), on the other hand, there is one largest node in each network, indicating that there is a major author of the paper on the subject of educational leadership, quality education and vocational education. This author collaborated with others to publish more papers than other authors.

Based on the same method, we also processed the data of the authoring unit, and finally obtained the cooperative network diagram shown in Figure 2. 


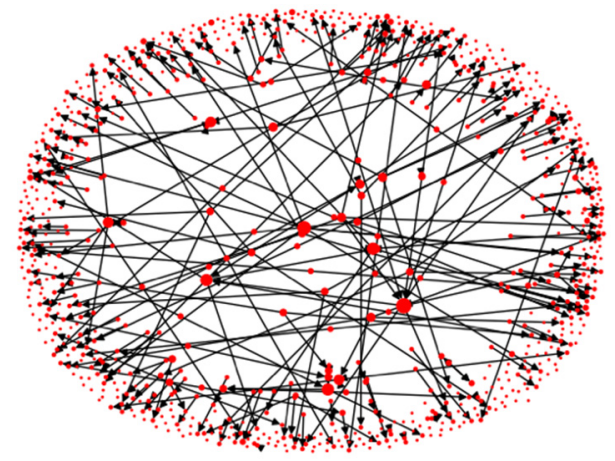

(a)

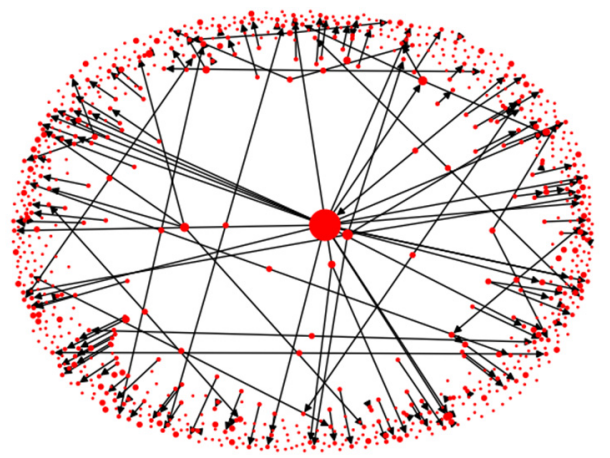

(c)

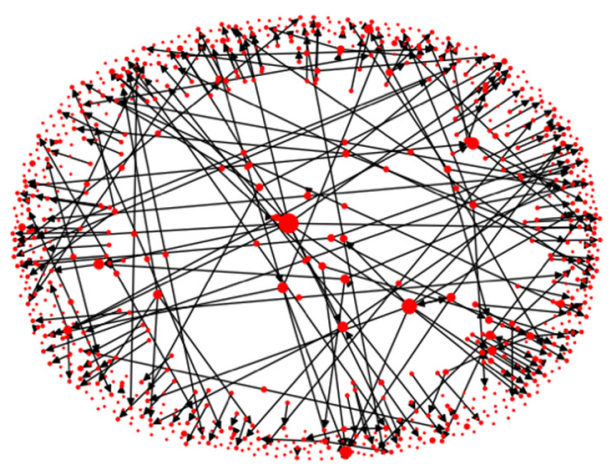

(b)

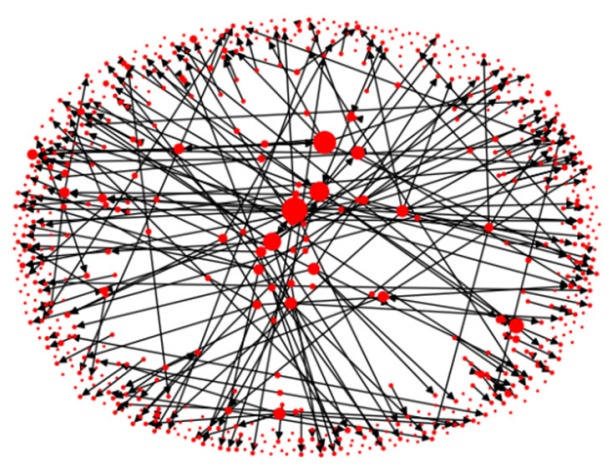

(d)

Figure 2. Cooperative Network Based on Author Unit

Among them, Figure (a) is the cooperation network diagram of the authors' units of the papers in the field of education leadership; Figure (b) is the cooperation network diagram of the authors' units of the papers in the field of education policy; Figure (c) is the cooperation network diagram of the authors' units of the papers in the field of quality education Figure (d) is a cooperative network diagram of the authors' units of the papers in the field of vocational education.

It can be seen from Figure 2 that the cooperation networks of the authors' units based on the four key words of education leadership, education policy, quality education, and vocational education have similar structures as in Figure 1. A large number of authors' units are independent, scattered around the network map, forming an elliptical scatter structure. The cooperation between the authors' units is obviously less than the cooperation between the authors of the papers, indicating that the collaborators are more likely to be reflected in the cooperation between the authors of the same unit. In Figure (c), the cooperation units are significantly less than those of the other three figures, and there is a large node, indicating that there is relatively little cooperation between different units in the quality education articles. At the same time, all cooperative papers have one most important cooperation unit. In Figures (a), (b) and (d), the numbers of papers collaborated by different units are significantly larger than the number of collaborated papers in Figure (c). The network structure of Figure (d) is more intensive, indicating that the authors of the articles of vocational education have more common cooperation across units.

\subsection{Comparative Analysis}

Through further analysis of Figures 1 and 2, we can find the following phenomena. The first is that there are so-called "key scholars" in different fields of education that is, when a researcher surpasses other researchers because of factors such as research ability, qualifications, academic status, and influence, he is more likely to receive attention. Thereby attracting more resources and getting more opportunities for cooperation, which is called the "Matthew effect." This makes such researchers have a large number of collaborators, but there is little cooperation 
between other collaborators, these researchers can be called "key scholars." From Figure 1, we can see that there are such so-called "key scholars" in the fields of educational leadership, quality education and vocational education. Further, as can be seen from Figure 2, there is also a so-called "key unit" based on the cooperation network structure of the authors' units, that is, the authors of different units have cooperation with the same author or different authors of a certain unit.

Secondly, according to the network structures we have given, no matter from the cooperation between the authors or the cooperation between the authors' units, there is no so-called independent schools, the aggregation phenomenon in the network structures.

Finally, we can see from the network diagram that there are a large number of independent authors and independent author units in all fields. Such papers account for more than $70 \%$ of the total, indicating that educators who publish papers generally lack cooperation with each other, which can be reflected in the independent scatter phenomenon in the network map.

\section{Conclusion}

This paper uses the method of the complex network to analyze the cooperative behavior of Chinese educational scholars publishing academic papers by using data on areas such as educational leadership, education policy, quality education, and vocational education crawled from cnki.net. Our conclusions show that most of the academic papers published by Chinese educational scholars are non-cooperative methods; among the authors of the co-authored papers, there is a significant "Matthew effect", that is, there are some key scholars in these fields, forming a typical cooperative network structure; third, there is no obvious aggregation effect between the authors of the co-published papers, indicating that there is a general and extensive connection between the collaborators.

The above conclusions provide valuable insights for us to understand the cooperative behavior of Chinese education scholars. This can provide educational cooperation for scholars and help the relevant departments to rationally allocate academic resources.

This paper is only based on the cooperative behavior of several educational fields. It used a simple cooperative network structure, did not analyze the ranking and influence of key authors, and did not further analyze the structure between collaborators. This will be our further work.

As researchers deep research on complex networks, in real-world networks, most of the nodes belong to multiple communities, that is, the community structure is "overlapping". For example, in the CPM (Clique Percolation Method algorithm proposed in 2005 , it is mentioned that there is a high possibility that a large complete subgraph will be formed due to the inner side of the community, but the association is unlikely. A complete subgraph appears, so the community can be found by finding factions in the network. The CPM algorithm is based on a complete subgraph algorithm, and multiple complete subgraphs are likely to share a node, so the CPM algorithm can discover the overlapping structure of the community (F. Reid, A. Mc Daid, \& N. Hurley, 2011). Whether there is such an overlapping community network among pedagogical researchers is also one of the directions of subsequent research.

\section{References}

Ahn Y., Bagrow J., \& Lehmann S. (2010). Link communities reveal multiscale complexity in networks. Nature, 466(7307), 761-764. https://doi.org/10.1038/nature09182

Barabasi A. L., \& Albert R. (1999). Emergence of scaling in random networks. Science, 286(5439), 509-512. https://doi.org/10.1126/science.286.5439.509

Barabasi A. L., \& Albert R. (1999). Emergence of scaling in random networks. Science, 286(5439), 378-382.

Barabási A L., Jeong H., \& Néda Z., et al. (2002). Evolution of the social network of scientific collaborations. Physica A., 311, 590-614. https://doi.org/10.1016/S0378-4371(02)00736-7

Bell L., \& Stevenson H. (2006). What is education policy? Education Policy, 23-40.

Gordon H. R. D. (2003). The history and growth of vocational education in America. Waveland Press, PO Box 400, Prospect Heights, IL 60070.

Haifeng Nie \& Jingjun Liu. (2014). The Emerging Small World of Chinese Economists. China Journal of Economics, 1(4), 107-139

Leithwood K. A., \& Riehl C. (2003). What we know about successful school leadership. Nottingham: National College 
for School Leadership.

Leithwood K., Jantzi D., \& Steinbach R. (1999). Changing leadership for changing times. McGraw-Hill Education (UK).

Merton R. K. (1968). The Matthew effect in science. Science, 159(3810), $56-63$. https://doi.org/10.1126/science.159.3810.56

Moody J. (2004). The structure of a social science collaboration network: Disciplinary cohesion from 1963 to 1999. American sociological review, 69(2), 213-238. https://doi.org/10.1177/000312240406900204

Reid F., Mc Daid A., \& Hurley N. (2011). Partitioning breaks communities. IEEE Computer Society, Kaohsiung, 102-109. https://doi.org/10.1109/ASONAM.2011.36

Smedt T. D., \& Daelemans W. (2012). Pattern for python. Journal of Machine Learning Research, 13(Jun), 2063-2067.

Team T. (2006). The Concept, Innovation and Related Theory of Quality-oriented Education. Educational Research, 2, 001 .

Uzzi B., \& Sprio J. (2005). Collaboration and Creativity: The Small World Problem. American Journal of Sociology, 111(2), 447-504. https://doi.org/10.1086/432782

Watts D J., \& Strogatz S. H. (1998). Collective dynamics of Small- World networks. Nature, 393(6638), 440-442. https://doi.org/10.1038/30918 